\title{
La transición epidemiológica de las y los adolescentes en México
}

\author{
José Ignacio Santos-Preciado, B Sc, M Sc, MD,(1) Juan Pablo Villa-Barragán, MC, MSP,(1) \\ Martha Angélica García-A vilés, MC, MSP, ${ }_{1}^{(1)}$ Graciela León-Álvarez, MC, MSP, EEA, (1) \\ Sonia Q uezada-Bolaños, MC, MSP, ${ }^{(1)}$ Roberto Tapia-Conyer, MC, MSP, MPH. . ${ }^{(2)}$
}

\section{Santos-Preciado JI,Villa-Barragán JP, García-Avilés MA, León-Alvarez GL, Q uezada-Bolaños S,Tapia-Conyer R. La transición epidemiológica \\ de las y los adolescentes en México. Salud Publica Mex 2003;45 supl 1:S140-S152.}

\section{Resumen}

Objetivo. Identificar los niveles de salud de la adolescencia en México en su demografía, contexto social y económico. Material y métodos. Se efectuó una revisión de la literatura y de estadísticas vitales, así como de encuestas de opinión, con la finalidad de evaluar el impacto de la transición epidemiológica frente a la disponibilidad y prioridades de los servicios de salud para adolescentes. El modelo epidemioló gico de 0 mran se utilizó para clasificar las características demográficas, sociales y económicas de adolescentes mexicanos como un esfuerzo para definir su impacto en la transición epidemiológica de este grupo de edad. Resulta dos. Los datos demográficos se presentan en una perspectiva de la transición epidemiológica, la cual nos permite realizar inferencias de las condiciones sociales, patrones de desarrollo y de necesidades en salud de este grupo poblacional en México. Los datos se presentan atendiendo a la demanda y disponibilidad de servicios de salud, el impacto de la educación, las oportunidades de empleo, el rol en la familia y el bienestar. 0 tros aspectos importantes presentados incluyen la educación sexual y la salud reproductiva, y los relacionados con el SIDA/VIH como pandemia en este grupo de edad. Conclusiones. Los hallazgos presentados revelan que la secuencia tradicional de variables, en la explicación de la transición epidemiológica, no puede se-

\author{
Santos-Preciado JI, Villa-Barragán JP, García-Avilés MA, \\ León-Alvarez GL, Quezada-Bolaños S, Tapia-Conyer R. \\ The epidemiologic transition \\ among adolescents in Mexico. \\ Salud Publica Mex 2003;45 suppl 1:S140-S152.
}

\begin{abstract}
A bstract
Objective. To identify the health status of adolescents in Mexico in their demographic, social and economic context. Material and Methods. A literature search and review of vital statistics, and opinion surveys were performed in order to asses the impact of the epidemiologic transition on the availability and priority of health services for adolescents. The 0 mran epidemiologic model was used to classify the demographic, social and economic characteristics of Mexican adolescents in efforts to define their impact on the epidemiologic transition of this age group. Results. D emographic data are presented in the perspective of the epidemiologic transition which permit us to make inferences on the social conditions, growth patterns and health needs of this age gro up in M exico. Data are presented regarding the demand and availability of health services, the impact of education and employment opportunities and the role of family and the economy on their well being. 0 ther important aspects presented include the role of education on the sexual and reproductive health of adolescents and the threat of the AIDS pandemic in this age group. Conclusions. These findings reveal a sequence of variables that can no longer be addressed in the traditional problem oriented approach and strongly suggest a need to profile the health needs of adolescents in an integrated,
\end{abstract}

(1) Centro N acional para la Salud de la Infancia y Adolescencia (CEN SIA). Secretaría de Salud, México.

(2) Subsecretaría de Prevención y Protección de la Salud. Secretaría de Salud, México.

Solicitud de sobretiros: Dr. José Ignacio Santos-Preciado. Centro N acional para la Salud de la Infancia y Adolescencia, Secretaría de Salud, México. Francisco P Miranda No 177. 06100 Col. Merced Gómez, México, D.F., México.

Correo electrónico: jisantos@ supernet.com.mx 
guir manejándose como hasta ahora y nos sugieren de manera imperativa la necesidad de perfilar las necesidades de salud de la adolescencia desde un enfoque holístico, con énfasis en la promoción de estilos de vida sana, que favorezcan un desarrollo justo y equitativo y que además ayuden a enfocar la respuesta social organizada.

Palabras clave: transición epidemiológica; adolescencia; salud; demografía; México holistic fashion with emphasis in health promotion and healthy life styles to favor their integral, just and equitable development, and also help focus the societal response in an integrated manner.

Key words: epidemiolo gic transition; adolescence; health; demography; Mexico
$E$ scribir sobre transición epidemiológica significa abordar concretamente tres variables que tienen relación con la salud: las demográficas, sociales y económicas. Es una aplicación inferencial y una interpretación del complejo salud-enfermedad y los determinantes demográficos, socioeconómicos y ecobiológicos de la dinámica poblacional. Se corre el riesgo de no identificar adecuadamente dimensiones de cambio, en tiempo, espacio o diversidad poblacional, en los patrones que determinan la aparición de la salud.

Con todo ello, en México este abordaje es urgente sobre todo para la aplicación de acciones esenciales de salud en un grupo de población, como es el de las y los adolescentes, que ancestralmente ha sido evadido en aspectos muy específicos de los efectos a la salud.

La teoría de la transición epidemiológica tiene como supuesto que la mortalidad sea un factor fundamental en la dinámica poblacional, aunada a la fertilidad como una significante covariable. Otro supuesto es que las enfermedades infecciosas en la transición epidemiológica van siendo desplazadas progresivamente por enfermedades degenerativas y otras producidas por el ser humano y sus estilos de vida.

En los modelos tradicionales de transición epidemiológica el eje del análisis lo constituye la mortalidad de la población; bajo este patrón, se distinguen tres escenarios: 1. La era de las epidemias y la peste, en la cual la mortalidad es alta y fluctuante, con una tasa alta de crecimiento poblacional, la esperanza de vida al nacer es baja y variable entre los 30 y los 40 años de edad. 2. La era del receso de las pandemias, en la que la mortalidad declina, las enfermedades infecciosas graves tienden a disminuir y la expectativa de vida aumenta. 3. La era de las enfermedades degenerativas y las provocadas por los estilos de vida; en ésta, la mortalidad y la fertilidad son bajas, las enfermedades crónico degenerativas, accidentes y violencias desplazan paulatinamente a las infecciones. ${ }^{1}$

La transición epidemiológica en las y los adolescentes en México, en el contexto de la población gene- ral, agrega una variable al modelo internacional: la polaridad social y económica. Muchos países comparten la polaridad social de uno como México, la coexistencia de índices de salud polares entre pobres y ricos establece una gran diferencia en la dotación de servicios de salud por parte del gobierno, el concepto de equidad es muy complejo, debido a la capacidad de respuesta ante necesidades diferenciales de la población. Hemos ubicado a México en el siguiente escenario: ante la disminución drástica de la mortalidad en el transcurso de 50 años existe un tránsito por una fase de alta polaridad entre las diferentes entidades del país y grupos sociales; el acceso a los servicios y el gasto en salud es diverso, así como la forma de presentación de los efectos; existe una gran necesidad de incorporar modelos de atención integrada con alta asociación entre los factores económicos y los efectos en salud. ${ }^{2}$

El modelo de transición epidemiológica en México queda definido por procesos sociales y de salud, así: 1) variables demográficas y patrones reproductivos; 2) el empleo y la educación; 3) la salud y la sexualidad, y 4) el acceso a los servicios en general. Con esta información es posible definir ante qué escenario en salud nos encontramos y poder ubicar, con la infraestructura en salud disponible, el modelo de atención más adecuado para adolescentes en México.

En este documento nos interesa organizar la información situacional de las y los adolescentes con una construcción que permita definir factores de riesgo, áreas de prioridad y patrones de actuación, con un enfoque de proyección hacia el futuro por lo que, inicialmente, se presenta información descriptiva, que es secundariamente analizada para priorizarla de acuerdo con las necesidades regionales, y sobre aspectos de salud-enfermedad, centrados en la mortalidad.

\section{Material y métodos}

Se realizó una búsqueda bibliográfica en documentos especializados, que permitieran tomar de manera consistente información estadística y resultados de en- 
cuestas actualizadas, para identificar el panorama epidemiológico, demográfico, social y económico, y relacionar estas dimensiones de la transición epidemiológica de las y los adolescentes en la dotación de servicios de salud. Los datos sobre aspectos demográficos fueron contrastados para complementar la caracterización de la población de adolescentes y construir el panorama de la transición epidemiológica. Los datos del Consejo Nacional de Población (CONAPO) se revisaron a la luz de la Encuesta Nacional de la Juventud, del Instituto Mexicano de la Juventud (IMJ), integrando los datos cuantitativos de la primera fuente con los datos cualitativos de la segunda. ${ }^{3,4}$

Información contenida en anuarios estadísticos de la Organización de Naciones Unidas (ONU) fue complementada con datos del Banco Mundial, asumiendo la clasificación económica de éste con las cifras de la ONU. Se tomaron un listado de 485 referencias bibliográficas, siete anuarios internacionales, de 1998 a 2001, seis Encuestas Nacionales de Adolescentes, el Censo Nacional de Población y Vivienda, encuestas del CONAPO y la Encuesta Nacional de Nutrición. Además, se acudió a la información de fuentes secundarias, provenientes de reportes de los servicios de los sectores de educación y salud.

Se analizaron los tamaños de muestra de las poblaciones en estudios inferenciales, se revisaron los objetivos de los estudios y el alcance de las afirmaciones y el grado de evidencia que aportaron a cada capítulo; con el equipo de trabajo se decidió la inclusión de la información que tuviera más consistencia y la mayor capacidad de ser generalizable a escala nacional, la cual finalmente quedó incluida en el contenido del presente documento.

Se tomaron como base los modelos epidemiológicos de Omram, ${ }^{1}$ para calificar la transición epidemiológica de adolescentes en México. A pesar de que la información sobre las causas de morbilidad tiene un amplio camino por consolidar y presenta dificultades para su identificación en el grupo de edad de 10 a 19 años, en el cual se tuvieron problemas muy importantes para tomar la decisión sobre aspectos de notificación de enfermedades, encontramos que en la mayoría de los informes de salud no se contempla para su estudio a la adolescencia dentro de los grupos de edad de $10 \mathrm{a}$ 14 y de 15 a 19 años.

Otro aspecto importante es que en casi la mitad de la información encontrada las agencias que publican los datos solamente ofrecen cuadros de salida, que en muchas ocasiones no correspondieron al análisis de nuestro estudio.

Por lo anterior, señalaremos algunas conclusiones analógicas a la información encontrada en fuentes se- cundarias que nos permitirán tener puntos de partida para la organización de acciones en salud desde el enfoque de la situación de la transición en las y los adolescentes.

\section{Resultados}

Para organizar los resultados de la información recabada fue necesario establecer diferentes variables que permitieran una revisión lógica y cronológica de los datos. Por lo que se tomaron en cuenta los siguientes puntos: aspectos demográficos, con un enfoque internacional, para aterrizar en México, estructura familiar, escolaridad, empleo, salud de los adolescentes, sexualidad y reproducción, fecundidad, factores de riesgo, morbilidad y mortalidad.

\section{Aspectos demográficos y económicos}

Existen en el mundo 6100 millones de personas que demandan servicios de alimentación, agua, salud, educación, vivienda, asimismo la satisfacción de necesidades como el vestido, entretenimiento, comunicación o esparcimiento. El consumo de bienes y servicios ha crecido mucho, de tal forma que esta variable no solamente define los patrones económicos sino también los estilos de vida; sin embargo, el acceso a éstos es diferencial, ya que la demanda de los servicios es alta y la oferta, o bien no existe o es de muy baja calidad. La satisfacción de las necesidades se ve entorpecida, sobre todo, por la disparidad en el desarrollo de las diferentes naciones. En el mundo, el ingreso per capita (IPC) más bajo es de 100 dólares (Etiopía), mientras que el más alto es de $\$ 38000$ (Suiza). México se encuentra en el lugar 33 con $\$ 4400$ dólares. El 75\% de la población está concentrada en las zonas más pobres del planeta, sin embargo $75 \%$ del consumo se encuentra en el otro $25 \%$ de la población. ${ }^{5}$

En el ámbito mundial, adolescentes y jóvenes (10 a 24 años de edad) representan la cuarta parte de la población; alrededor de 1700 millones de personas se encuentran en este grupo de edad, de los cuales $85 \%$ viven en los países en desarrollo.

En México la población de este grupo de edad se ha incrementado considerablemente a partir de la segunda mitad del siglo XX en números absolutos. En 1950, la población total de México era cerca de 25.7 millones de personas y la población adolescente y joven era de casi ocho millones, la cual representaba alrededor de $31 \%$ de la población total (22\% la población adolescente y $9 \%$ la población joven). ${ }^{6}$

De acuerdo con el censo 2000, en México 21.3\% de la población es adolescente. Viven 29.7 millones de 
adolescentes y jóvenes (20.7 millones de adolescentes entre 10 y 19 años de edad; 9 millones de jóvenes de 20 a 24 años de edad). En cuanto a la proporción de adolescentes en el resto de la población hay poca variación de 1950 a la fecha; para este año los adolescentes representaban $22.23 \%$ de la población total; en 1980 fue de $25 \%$, y para el año 2000, 21.26. Sin embargo, el incremento en números absolutos ha sido de casi $250 \%$. Esto significa que si en 1950 había alrededor de ocho millones de adolescentes y jóvenes, para el año 2000 tenemos cerca de 30 millones (70\% adolescentes y 30\% jóvenes). ${ }^{7-9}$

Este incremento de la población joven llevó a que 1985 se proclamara por la ONU el Año Internacional de la Juventud. A partir de este momento, los Estados empezaron a impulsar con más fuerza investigaciones sobre las diversas problemáticas que atañen a adolescentes y jóvenes, como son: el acceso a la educación por parte de todo adolescente sin importar su condición económica y su condición de género, la fuerza de trabajo en potencia y los problemas futuros como la mala calidad del empleo y el desempleo, las adicciones y sus consecuencias para la salud futura, los mitos y tabúes sobre su salud sexual y reproductiva y sus consecuencias en la morbilidad y mortalidad de éstos, y el enorme problema de la violencia, entre otros. ${ }^{10}$

La proporción de población joven aumenta a medida que disminuye el IPC de los países $(r=-92)$. Por ejemplo, en Alemania 18\% son adolescentes y en Bangladesh, $47 \%$ del total de la población. Aparentemente tiene una correlación perfecta con el IPS de la pobla- ción, por lo que la población proporcional (relación entre menores y mayores de 15 años de edad), tiende a ser más alta a medida que los países son más pobres; este factor económico debe resaltar el hecho de la reproducción lineal de la pobreza, por lo que los índices de polaridad, ricos: pobres, cada vez tienden a extender la brecha. En el cuadro I se presentan nueve indicadores internacionales que clasifican a los países de acuerdo a su IPC. ${ }^{11-13}$

Para el año 2050, la población general habrá aumentado 50\%, es decir, el número de personas será de alrededor de 9300 millones. Para este tiempo 85\% de la población se concentrará en los países más pobres, quienes presentan ya amplia problemática para la dotación de servicios y empleos. El ritmo mundial de crecimiento de la población es de 1.3\% (77 millones de nacimientos al año). Sin embargo, las tasas de crecimiento no son las mismas para todos los países. De mantenerse las tasas de crecimiento actuales, tendremos entonces una distribución del crecimiento con una variación muy alta. Por ejemplo, hay países de Europa Oriental, como Croacia y Bulgaria, que tienen tasas de crecimiento negativas ( -0.8 y -0.7 respectivamente). Los países de la Unión Europea apenas habrán aumentado su población, pues tienen en promedio una tasa de crecimiento de 0.4 . Sin embargo, muchos países asiáticos y latinoamericanos que tienen tasas de crecimiento que van de 1.2 a 1.8, como Argentina, México, Brasil, Chile, Irán, Indonesia, Perú, Singapur, duplicarán su población para el año 2050. Y otros tantos como Colombia, Rwanda, Sudáfrica, Venezuela, Bolivia, Nepal,

\section{Cuadro I}

INDICADORES DEMOGRÁFICOS BÁSICOS DE PAíSES SEGÚN INGRESO PER CAPITA*

\begin{tabular}{|c|c|c|c|c|}
\hline Indicadores & $\begin{array}{c}\text { Países con ingreso } \\
\text { bajo }\end{array}$ & $\begin{array}{l}\text { Países con ingreso } \\
\text { ingreso bajo }\end{array}$ & $\begin{array}{l}\text { Países con ingreso } \\
\text { medio alto }\end{array}$ & $\begin{array}{l}\text { Países con ingreso } \\
\text { alto }\end{array}$ \\
\hline Población total & $2.5 \mathrm{~mm}$ & $2 \mathrm{~mm}$ & 638.4 millones & 902.9 millones \\
\hline Crecimiento anual (\%) & 1.9 & 0.9 & 1.3 & 0.7 \\
\hline Esperanza de vida al nacer (años) & 58.9 & 69.5 & 69.7 & 78.1 \\
\hline Tasa de fecundidad (nacimientos por mujer) & 3.6 & 2.1 & 2.3 & 1.7 \\
\hline Tasa de mortalidad infantil (x 1000 recien nacidos vivos) & 76.1 & 32.6 & 27.9 & 5.7 \\
\hline Población urbana (\%) & 31.9 & 42 & 75.9 & 78.8 \\
\hline Analfabetismo femenino (\%) & 47 & 21.5 & 11.9 & - \\
\hline Analfabetismo masculino (\%) & 28.3 & 9.2 & 8.4 & - \\
\hline Densidad de población (x km²) & 75.6 & - & - & 29.2 \\
\hline
\end{tabular}

* Clasificación del Banco Mundial, de acuerdo con el ingreso económico. Los países con ingreso bajo tienen un ingreso per capita menor 0 igual a $\$ 755$ dólares; el ingreso medio bajo va de \$756 a \$2 995 dólares; el ingreso medio alto de $\$ 2996$ a \$9265. El ingreso alto es de $\$ 9266$ y más dólares 
Nigeria, triplicarán su población (con tasas de crecimiento de 2 a 2.8). Los países con tasa superiores a 3.0 quintuplicarán su población en este mismo periodo. ${ }^{13}$

Este comportamiento se debe a que el conjunto de la población ha crecido de manera homogénea. La variación más importante ha sido en el porcentaje que representan los niños y los adultos en el total de la población. Algunas explicaciones de esto se encuentran en el descenso de la fecundidad y en el número de hijos por mujer. Las poblaciones adolescente e infantil tienen una tendencia a la disminución; en los próximos años, representarán cada vez menor proporción entre la población total, pero la cifra absoluta seguirá siendo considerable. ${ }^{8}$

El efecto en el futuro será una menor proporción de población económicamente activa, que tendrá una carga social cada día mayor y que requerirá tener mejor condición productiva y mayor ingreso per capita que los adultos actuales. La población dependiente se redujo de $47.5 \%$ en 1970 a $31.9 \%$ en 2002; el grupo de 15 a 64 años de edad se incrementó de 48 a 63\%, y el grupo de mayores de 64 años de edad aumentó un punto porcentual. ${ }^{14}$

La población de adolescentes se concentra en medianas y grandes urbes, encontrando que $57 \%$ de ellos se encuentra en ocho entidades de la República Mexicana con áreas urbanas más densas (Bono poblacional). ${ }^{14}$ En otro enfoque, $49.9 \%$ de jóvenes se encuentran en siete de las 32 entidades federativas del país. La juventud en México es predominantemente urbana (localidades $>2500$ habitantes) en donde se ubica $60.8 \%$ del total, proporción más alta que la población total.

Las y los adolescentes incrementan en número absoluto continuamente, sin embargo, su proporción con respecto al total de la población tiende a la baja, debido a la disminución de la tasa de fecundidad, así como al efecto del crecimiento económico y de los programas de planificación familiar y de la migración continua de la población desde poblaciones menores de 15000 habitantes hacia zonas urbanas en la búsqueda de mejores oportunidades sociales y económicas.

El reto social que implica este panorama demográfico es el de garantizar la satisfacción de las necesidades básicas de esta población, y crear las condiciones suficientes para el desarrollo de sus capacidades y planes de vida para el futuro. ${ }^{14}$

\section{Estructura familiar}

En México entre 35 a 40\% de adolescentes viven en hogares de extrema pobreza. La gran mayoría viven en familias con madre y padre y $26.6 \%$ han salido del ho- gar paterno. Entre adolescentes de 15 a 19 años de edad, $1.5 \%$ son jefes de familia (hombres $2.3 \%$ y mujeres $0.7 \%$ ). El $63.9 \%$, del total de jóvenes en este mismo grupo de edad viven en hogares nucleares y en hogares extensos, 33.2\%; de los hogares extensos, 30 de cada 100 son monoparentales y, de las familias nucleares, son monoparentales solamente $14 \%$.

El CONAPO identifica al menos 12 tipos de grupos familiares y $22 \%$ de la población de adolescentes viven en hogares monoparentales con prevalencia de la madre. Aunque los jóvenes en México viven solos con una frecuencia baja (1.1\%) esto en el área urbana es cuatro veces más frecuente que en la rural, lo que significa que las y los adolescentes en México salen de sus casas con la intención directa de vivir en pareja $o$ en asociación con otras personas.

En cuanto al estado civil de las y los adolescentes, existen más mujeres solteras menores de 14 años de edad en áreas urbanas (95\%), que en áreas rurales (77\%). Entre adolescentes de 10 a 15 años de edad 99\% son solteros y en los de 16 a 20 años de edad, hasta uno de cada 10 se encuentran ya en unión; entre las mujeres este porcentaje aumenta hasta tres veces más con relación a los hombres, en este mismo grupo de edad. ${ }^{3,4}$

\section{Escolaridad}

La Encuesta Nacional de la Juventud (ENJ) señala que entre los 12 y los 14 años de edad no acuden a la escuela $11.6 \%$ de adolescentes; de los 15 a los 19 años de edad no acuden $41.3 \%$, y que al llegar a los 19 años de edad más de $75 \%$ de jóvenes ha abandonado la escuela por motivos económicos y falta de acceso en su localidad, principalmente.

El promedio de escolaridad en México es actualmente de 7.7 grados, cerca de tres veces más que en 1960. Se prevé que en una década más el promedio será de nueve grados. La desigualdad en los niveles de escolaridad alcanzados entre la población joven que reside en localidades rurales respecto a la que reside en las ciudades es de 6.5 y 9.2 años, respectivamente.

En México 97\% de las y los adolescentes saben leer y escribir, la población general ha incrementado su nivel de instrucción y la brecha entre hombres y mujeres es nula, no así entre adolescentes indígenas en los cuales el analfabetismo puede llegar a 9\% entre las mujeres. El problema de la asistencia a la escuela es la necesidad de preparación más especializada en los centros de trabajo. ${ }^{3,4}$

En México solamente 53\% de la población menor de 19 años de edad asiste a la escuela; de los jóvenes de 10 años de edad acude $95 \%$, pero de los mayo- 
res de 15 años de edad solamente 17\%. En México, cuando los adolescentes cumplen 19 años de edad, han abandonado la escuela cerca de $89 \%$ de ellos. ${ }^{3,4}$

\section{Empleo}

En la actualidad los adolescentes forman una parte importante de la población económicamente activa. Cada año se agregan al mercado de trabajo personas menores de 20 años de edad y para el año 2000 los hombres menores de 19 años de edad que participan ya en el campo laboral son cerca de $44 \%$ del total de ellos y las mujeres $24 \%$ de ellas, en total 35.8. De la población económicamente inactiva de adolescentes $56 \%$ se dedica a estudiar, a labores domésticas $22 \%$; entre las mujeres que no estudian $32.7 \%$ se dedican a labores del hogar, mientras que de los hombres lo hacen solamente $1.1 \%$ (cuadro II). ${ }^{15}$

La tasa de participación en el campo laboral varía de acuerdo con la edad, sin embargo, es de notar que $8 \%$ de adolescentes de 12 a 14 años de edad ya se encuentran en el mercado de trabajo siendo esto más notorio en los hombres. En adolescentes de 15 a 19 años de edad la tasa de participación promedio es del $35 \%$ pero sigue siendo 2:1 hombre mujer la razón de participación.

El que los adolescentes participen en los procesos productivos tiene implicaciones diversas en cuanto a la calidad del trabajo que asumen. La calidad del trabajo desde nuestra perspectiva de salud debe contar con los siguientes atributos: tener jornadas de trabajo acordes con la edad del sujeto, contar con un salario equitativo, tener derecho a la seguridad social, tener normas básicas de seguridad e higiene acordes con la ley, y contar con prestaciones adicionales.

Otra implicación del trabajo en adolescentes es que quienes se agregan a la planta laboral en nuestro país

\section{Cuadro II \\ ACtividad PRINCIPAL QUE REALIZA La POBLACIÓN ECONÓMICAMENTE INACTIVA ADOLESCENTE Y JOVEN. México 2000}

\begin{tabular}{|c|c|c|c|c|c|c|c|}
\hline \multirow{3}{*}{$\begin{array}{l}\text { Grupos } \\
\text { de edad } \\
\text { Total }\end{array}$} & \multirow{3}{*}{$\begin{array}{l}\text { PEI* } \\
63.5\end{array}$} & \multicolumn{3}{|c|}{ Estudiar } & \multicolumn{3}{|c|}{ Labores domésticas } \\
\hline & & Total & Hombre & M ujeres & Total & Hombre & M ujeres \\
\hline & & 56.1 & 70.9 & 46.5 & 22.1 & 0.9 & 35.8 \\
\hline 12-14 años & 90.3 & 79.0 & 81.5 & 76.6 & 4.2 & 0.4 & 7.8 \\
\hline 15-19 años & 64.4 & 55.6 & 67.5 & 47.5 & 20.0 & 1.1 & 32.7 \\
\hline $0-24$ años & 43.5 & 23.5 & 48.6 & 15.6 & 51.7 & 1.9 & 67.3 \\
\hline
\end{tabular}

Fuente: IN EGI, X II Censo N acional de Población y Vivienda 2000 lo hacen por problemas económicos de la familia y la sociedad, lo que es seguido del abandono escolar, además de la forma en la que ellos se desempeñan cuando son económicamente inactivos. ${ }^{14}$

\section{La salud de adolescentes}

Servicios de salud

El acceso a los servicios de salud en la población es medido a través de la derechohabiencia $(\mathrm{DH})$ a la seguridad social. Entre la población general la DH es de $40 \%$ para todo el país, aunque es de señalar que para los niños de menos de 10 años de edad disminuye a $37.3 \%$ y para adolescentes de 10 a 19 años de edad es tan sólo de $35.6 \%$. Entre hombres y mujeres no existe diferencia para esta observación. De adolescentes el Instituto Mexicano del Seguro Social (IMSS) asegura solamente a $28.4 \%$ y el Instituto de Seguridad y Servicios Sociales de los Trabajadores del Estado (ISSSTE) a $5.6 \%$, porcentajes inferiores a aquellos de la población general.

Como ya se comentó, la DH en la República Mexicana es de $37.3 \%$, siendo las entidades federativas con mayor proporción de $\mathrm{DH}$ los estados de Coahuila (63.7), Nuevo León (56.4), Baja California Sur (56.4); y los estados con más baja DH, Chiapas, Guerrero y Oaxaca (17.6, 20.3, 22.6\%, respectivamente); situación similar ocurre con la DH de adolescentes en todo el país la cual es de $35.6 \%$ y las cuatro entidades con mayor proporción van desde 66 hasta $55.4 \%$; y las de más baja proporción van de 15.5 hasta $21.5 \%$. Se destaca cómo la polaridad en el acceso a la seguridad social es muy considerable; aunque la proporción de adolescentes incluidos en el campo laboral tiene variaciones menores. En los estados donde la $\mathrm{DH}$ es más baja por alguna razón, el ISSSTE incrementa sus coberturas de $5 \%$ a escala nacional, hasta $7 \%$ en estas entidades.

No existe ninguna estadística confiable que nos permita conocer la intensidad de uso de los servicios de salud en sus diferentes modalidades por parte de adolescentes; solamente el Sistema de Información en Salud para Población Abierta 2000 nos permite apreciar que $7 \%$ de toda la consulta externa de primer nivel de atención nacional es demandada por población de entre 10 y 19 años de edad. ${ }^{8}$

Sexualidad y reproducción

La Organización Mundial de la Salud (OMS) (1975) define la salud sexual como la integración de los elementos somáticos, emocionales, intelectuales y socia- 
les del ser sexual, por medios que sean positivamente enriquecedores y que potencien la personalidad, la comunicación y el amor. ${ }^{16}$

En cuanto a la salud sexual y reproductiva, la edad promedio de la iniciación de la vida sexual activa es de 15.4 años; para el área rural 13.8 y para el área urbana 16.7. ${ }^{4}$ Existe una correlación directa entre el promedio de escolaridad y el inicio de la vida sexual activa. ${ }^{3}$ Como resultado de estas prácticas, ocurren más de 25000 embarazos anuales en madres menores de 19 años de edad, que terminan en aborto y constituyen cerca de $10 \%$ del total de los embarazos.

El uso de método anticonceptivos, en general, se ha incrementado hasta en $22 \%$ o más, ${ }^{14}$ sin embargo, entre jóvenes y adolescentes estas cifras son menores y con prevalencia del uso del preservativo. La diferencia entre área rural y urbana en ocasiones es hasta de 20 puntos porcentuales, sobre todo en estados como Oaxaca y Guerrero; la demanda insatisfecha en el área urbana es hasta de $8 \%$ y en el área rural hasta de $22.2 \% .^{14}$

Existe una correlación estrecha entre la tasa de fecundidad y la prevalencia del uso de métodos anticonceptivos (Guerrero 3.2 de tasa de fertilidad versus $52 \%$ uso de anticonceptivos; Baja California, 2.1 de fertilidad versus $77 \%$ de uso de anticonceptivos). ${ }^{14}$

Entre la población adolescente se estima que durante el año 2000 ocurrieron en el país cerca de 366000 nacimientos en madres de menos de 19 años de edad, lo que representa $17 \%$ del total de nacimientos y una tasa específica de fecundidad de $70.1 \times$ mil mujeres de ese grupo de edad. A pesar de que durante los últimos seis años el número de nacimientos se redujo en poco más de 10\%, la prevención del embarazo no planeado en las adolescentes continúa siendo un desafío prioritario en salud reproductiva. ${ }^{17}$

Adicionalmente, la demanda insatisfecha entre las mujeres unidas de 15 a 19 años de edad es la más alta de todos los grupos etáreos y representa más del doble del valor estimado para el resto de las mujeres.

\section{Fecundidad}

La fecundidad de las mujeres de 15 a 19 años de edad ha descendido en México desde la década de los setenta. La proporción de mujeres de 15 a 19 años de edad que procrea al menos un hijo (a) disminuyó de poco más de una de cada siete, en 1975, a una de cada doce en 1990, y a una de cada catorce en 1999. En 1975, las adolescentes registraban una tasa de fecundidad de 130 nacimientos por mil mujeres, valor que disminuyó a 81 en 1995, y a 72 por mil en 1999. Sin embargo, la velocidad de descenso de la fecundidad de este grupo no ha sido tan rápida como en los otros segmentos de edad. Por ello, su contribución a la tasa global de fecundidad ha venido creciendo, al pasar de $11.1 \%$ en 1975 a 14.5 en 1999.

El embarazo en las adolescentes es aún preocupante, no sólo por los riesgos que tiene una fecundidad precoz para la salud de la madre y de su descendencia, sino también porque las pautas de procreación en la adolescente pueden limitar las oportunidades de su desarrollo personal. ${ }^{8}$

\section{Indice de desarrollo relativo al género}

El índice de desarrollo relativo al género (IDG), que utiliza las mismas dimensiones que el índice de Desarrollo Humano (IDH), es decir: esperanza de vida al nacer, logro educacional y producto per capita pero introduce un ajuste para capturar las desigualdades de género. Este indicador permite aproximarse a la medición de las asimetrías entre los sexos y permite apreciar las posibilidades diferenciadas de hombres y mujeres en el desarrollo de sus capacidades y potencialidades.

El último informe del Programa de Naciones Unidas para el Desarrollo (PNUD) sobre desarrollo humano calculó el IDG de un total de 144 países. México ocupó la posición 50 en la clasificación mundial, con un IDG 0.775. En 1997 se realizó un análisis del IDG de las 32 entidades federativas del país, identificándose que el IDG es inferior al IDH en todas ellas, lo que revela que el progreso de las mujeres en materia de desarrollo humano está a la zaga del registrado por los hombres. Situación que debe considerarse en el proceso evolutivo de las y los adolescentes. ${ }^{3}$

\section{Factores de riesgo}

Entendiendo el factor de riesgo como una característica o circunstancia cuya presencia aumenta la posibilidad de que se produzca un daño o resultados no deseados, las y los adolescentes por diversas circunstancias ambientales, familiares e individuales, frecuentemente desarrollan conductas que participan como factores de riesgo.

Las conductas de riesgo, que a su vez pueden constituir daños más comunes son: adicciones, (tabaquismo, alcoholismo y drogadicción), exposición a ambientes peligrosos y violentos, que asociados potencializan la probabilidad de que las y los adolescentes sufran accidentes, suicidios y homicidios, entre otros.

Otras conductas de riesgo importantes son las relaciones sexuales sin protección, que pueden llevar a infecciones de transmisión sexual como el VIH/SIDA, 
y también a embarazos no planeados. También la mala alimentación, que predispone a desnutrición u obesidad. ${ }^{8}$

La falta de información veraz y oportuna en el marco de una educación sexual con enfoque de género, incluyendo masculinidades y femineidades, al no existir genera comportamientos de riesgo, como los accidentes automovilísticos, heridas por arma de fuego, falta de percepción de riesgo para adquirir algunas infecciones de transmisión sexual (ITS) o para provocar un embarazo no planeado, consecuencia ésta generada por no usar protección (condón femenino o masculino), por no tener sexo seguro (sexo no penetrativo $)^{4}$. De igual forma, muchos de estos embarazos terminan en abortos inducidos, con los consecuentes efectos en la fertilidad futura de la adolescente.

La epidemia del SIDA en México es predominantemente sexual, toda vez que este tipo de transmisión ha sido la causante de casi $90 \%$ de los casos acumulados de SIDA. La epidemia se concentra fundamentalmente en el grupo de $\mathrm{HSH}$, con poco más de $50 \%$ del total de casos acumulados, pero con una tendencia ascendente, en los últimos años, en los casos de tipo heterosexual. ${ }^{24}$

La relación de casos de SIDA entre hombres y mujeres es de 6 a 1 , en tanto las personas de 25 a 34 años de edad constituyen el grupo más afectado, con $41.6 \%$ de los casos registrados en adultos. ${ }^{24}$

En 1999, el SIDA ocupó el lugar 16 como causa de muerte, con una tasa de 4.3 por cada 100 mil habitantes. Sin embargo, la población más afectada por la epidemia ha sido la población de 25 a 34 años de edad, en la que el SIDA representó la cuarta causa de muerte en hombres y la séptima entre las mujeres. ${ }^{24}$

Existe evidencia de que el manejo adecuado de las ITS disminuye en 50\% la transmisión sexual del VIH. Por tal motivo, todas las ITS se incluyeron, desde 1997, para integrar lo que hoy se conoce como el Programa Nacional para la Prevención y Control del VIH/SIDA e ITS. La OMS estimó que en México ocurren anualmente siete millones de casos nuevos de ITS curables: tricomonas (3.39 millones de infecciones), clamidia (1.92 millones), gonorrea (1.36 millones) y sífilis $(0.24$ millones). ${ }^{24}$

El riesgo que suponen las relaciones sexuales, sin protección, para las y los jóvenes queda de manifiesto en las tasas elevadas de ITS y embarazos no planeados. La iniciación temprana de sus relaciones sexuales representa un factor de riesgo adicional, ya que la tasa de cambio de pareja sexual es más elevada entre las y los jóvenes de 15 a 24 años de edad. ${ }^{8}$

La prevención de las ITS y de los embarazos no planeados constituye una parte fundamental de la sa- lud sexual, por lo que la información clara y precisa de cómo evitar estos riesgos debe ser accesible a toda la población. Se ha demostrado que la utilización de anticonceptivos y condones es más constante si esta información se recibe antes de la primera relación sexual, es decir, en la preadolescencia, además de que no promueve el inicio más temprano de las relaciones sexuales. ${ }^{18,19}$

En ausencia de vacunas efectivas contra las ITS, incluyendo el SIDA, la forma efectiva para evitar el riesgo de infecciones es el uso del condón. El uso correcto y constante del condón protege entre 90 a 95\% de la transmisión de ITS, incluyendo el SIDA. Aunque existen evidencias de incremento del uso del condón, especialmente entre HSH y trabajadoras del sexo comercial, para controlar el crecimiento de estas infecciones es urgente aumentar el uso adecuado del condón en otras poblaciones, especialmente entre las y los jóvenes. ${ }^{18}$

\section{Salud Mental}

Los problemas de salud mental se han incrementado drásticamente en las últimas décadas; los datos disponibles indican que los problemas mentales están entre los que contribuyen a la carga global de enfermedades y discapacidades. Los niños y adolescentes, entre otros, constituyen un grupo que vive en condiciones o circunstancias difíciles que los ponen en riesgo de ser afectados por algún trastorno mental. Se reporta que la depresión, los intentos suicidas y la ansiedad, se encuentran entre los trastornos más frecuentes. Asimismo, la falta de escolaridad ha desencadenado factores precipitantes de conductas antisociales. ${ }^{20}$

\section{Suicidio}

El suicidio en adolescentes adquiere cada vez mayor interés para los profesionales de la salud, y el reconocimiento de los factores de riesgo asociados, de las opciones de tratamiento y de las estrategias de prevención se revelan como aspectos esenciales en el manejo global. Son más los adolescentes que las adolescentes que logran morir, pero son más las adolescentes que lo intentan. Se ha identificado que tras cada suicidio conocido hay 50 intentos que no se logran detectar y, por supuesto, no se toma ninguna medida de apoyo para los que lo realizan. En 1989, Stillion, Mc Dowell y May propusieron un modelo de la trayectoria del suicidio, que comprende cuatro categorías de factores de riesgo que contribuyen al pensamiento suicida: los aspectos biológicos, los psicológicos, los cognitivos y los ambientales que pueden influir en la idea suicida, ya sean 
directamente o afectándose entre sí para dar el comportamiento autodestructivo. ${ }^{21}$

Durante el periodo de 1970 a 1994, la tasa de suicidios en ambos sexos pasó de 1.13 por 100000 habitantes en 1970 a 2.89 por 100 000; en 1994 aumentó $156 \%$, con mayor fuerza para la población masculina. ${ }^{21}$

En términos de la mortalidad proporcional, el suicidio pasó de 0.11 a $0.62 \%$ de todas las defunciones.

Los porcentajes de variación más elevados en la tasa de mortalidad por suicidio se observaron en las poblaciones de mayor edad (más de 65 años) y en la más, joven (menor de 19 años).

En el grupo de edad de 15 a 24 años el suicidio en términos absolutos es raro, pero desde mediados del siglo tiene una tendencia a aumentar paulatina y progresivamente, pasando a constituir un problema de salud pública. ${ }^{21}$

\section{Adicciones}

Según datos de la Encuesta Nacional de Adicciones (ENA) de 1998, 10\% de las y los adolescentes fuman y, de acuerdo con datos del Consejo Nacional Contra las Adicciones (CONADIC), actualmente se registran 122 defunciones diarias en la población en general, relacionadas con el consumo de tabaco. Existen 14 millones de fumadores, de los cuales más de $70 \%$ se iniciaron antes de los 14 años de edad, y el universo de fumadores pasivos es de 48 millones de personas. El 75\% de los estudiantes empezaron a fumar antes de los 15 años de edad, siendo el promedio de 13 años para los hombres y 14 años para las mujeres. El 9\% de los hombres y $4 \%$ de las mujeres empezaron a fumar antes de los 11 años de edad. ${ }^{20}$

El consumo de drogas ilegales también es común. Se calcula que alrededor de $4 \%$ de los hombres de entre 12 y 17 años de edad han probado alguna droga en su vida. Las drogas consumidas con mayor frecuencia son los inhalables y la marihuana y, en menor proporción, la cocaína y las pastillas psicotrópicas. El 40\% de la población reporta que en su familia se consumen alcohol y otras drogas. En el bachillerato, $7 \%$ de la población reportó haber fumado marihuana en los seis meses anteriores y $2 \%$ ya había probado la cocaína (figura 1$)^{20}$

Entre 1976 y 1997, en los estudiantes de educación media y media superior, se observa un incremento importante en el consumo de la cocaína, pasando de $0.5 \%$ a $4 \%$, respectivamente. El consumo de marihuana el año anterior a la encuesta fue de $3.2 \%$ y el de cocaína de $2.7 \% .^{20}$

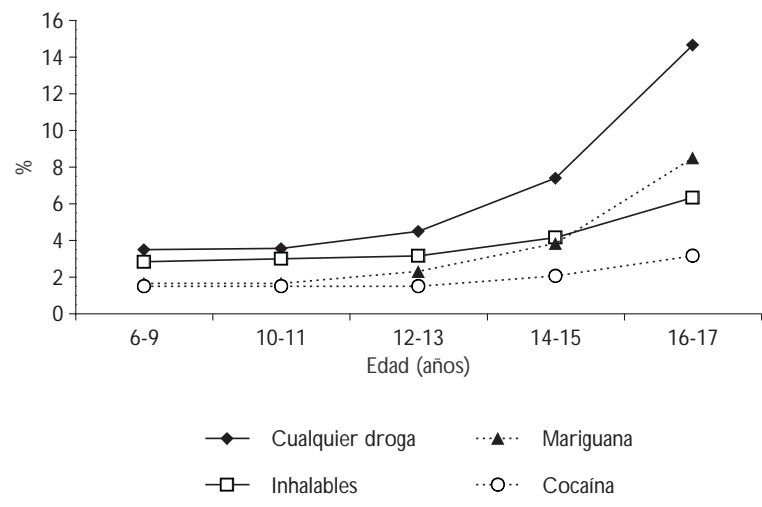

Figuras 1. Consumo de SUStancias adictivas ILEgALES en Adolescentes Menores de 17 años de edAd. MéXico, 2000

\section{Nutrición}

Las necesidades de nutrimentos durante la adolescencia se incrementan por existir aumento de la tasa de crecimiento y cambios en la composición corporal, que son diferentes para cada sexo. La Encuesta Nacional de Nutrición 1999 arroja los siguientes resultados, de acuerdo con los grupos de edad estudiados y que incluyen a la población adolescente: en el grupo escolar (5-11 años de edad) existe una elevada prevalencia de sobrepeso en la población. Una de cada cinco personas de este grupo poblacional presentan sobrepeso $\mathrm{u}$ obesidad, los que se presentan con mayor frecuencia en las zonas urbanas. Otro problema importante en este grupo de edad es la anemia, con una prevalencia en el ámbito nacional similar a la de sobrepeso y obesidad. Se encontraron consumos dietéticos deficientes de vitamina A y zinc, y consumos adecuados o elevados de proteínas y ácido fólico. Los resultados de las determinaciones de micronutrimentos indican deficiencias importantes de hierro, vitaminas A y zinc, y consumos adecuados o elevados de proteínas y ácido fólico.

Los resultados de las determinaciones de micronutrimentos indican deficiencias importantes de hierro, vitamina $E$, vitamina $C$, zinc y vitamina $A$, y en menor medida, de ácido fólico. En resumen, en este grupo de población los principales problemas de nutrición son el sobrepeso, la anemia y la deficiencia de varios micronutrimentos.

En el grupo de mujeres en edad fértil (12 a 49 años de edad) que incluye a la población adolescente me- 
nor de 19 años de edad, los hallazgos de la encuesta mostraron prevalencias elevadas de sobrepeso y obesidad en mujeres en edad fértil. Otro problema importante fue la prevalencia de anemia. Se encontraron consumos dietéticos deficientes de zinc, vitamina A, hierro, folato y vitamina $C$.

Los resultados de las determinaciones bioquímicas de micronutrimentos indican deficiencias importantes de hierro, zinc y vitaminas $\mathrm{E}$ y $\mathrm{C}$ y, en menor medida, deficiencia de ácido fólico. No se encontró deficiencia importante de vitamina A. ${ }^{22}$

\section{Daños a la salud}

Las causas más importantes de mortalidad en adolescentes de 10 a 14 años de edad son los accidentes y los tumores, así como las enfermedades congénitas. Entre adolescentes de 15 a 19 años de edad, la muerte es igualmente por accidentes y violencias, tumores en menor cuantía y epilepsia. Entre las causas accidentales y violentas figuran como principales componentes los accidentes de tránsito y el suicidio; entre los tumores, el linfoma y la leucemia. Estas causas cuentan con pocos recursos asignados para su tratamiento $y$, sin embargo, constituyen más de $80 \%$ de los casos de muerte que son prevenibles.*

\section{Morbilidad}

Los anuarios de morbilidad señalan que en el periodo de 1990 a 1999 las primeras cinco causas de enfermedad en el grupo de 5 a 14 años de edad fueron: infecciones respiratorias agudas, infecciones intestinales mal definidas y amibiasis; a partir de 1995, el rubro "otras helmintiasis" ocupa el cuarto lugar, y la ascariasis el quinto.

El siguiente grupo de edad corresponde al periodo de los 15 a los 24 años, en el cual las cinco primeras causas de enfermedad fueron: infecciones respiratorias agudas, otras infecciones intestinales mal definidas, la amibiasis y otras helmintiasis; el quinto lugar lo ocupa la otitis media aguda.

A pesar de que las ITS y la infección por el VIH/ SIDA no ocupan los primeros lugares de morbilidad, de acuerdo con el Sistema de Vigilancia Epidemiológica, al 31 de diciembre de 1998 se habían reportado más de 4500 casos de SIDA entre la población de 15 a 24 años de edad, de los cuales 626 tenían entre 15 y 19 años al momento del registro.

\footnotetext{
* Villa-Barragán JP. Priorización de necesidades de salud en México. 2002. Documento mimeografiado.
}

Se acepta que existe subregistro en la notificación de ITS, a pesar de ello se conoce que la mayor frecuencia de infecciones de transmisión sexual en 1999 correspondió a la candidiosis urogenital, con una tasa de 136.4 casos por 100000 habitantes, y la tricomoniasis urogenital, con 108.8 por 100000 .

El reconocimiento de que las y los adolescentes en México tienen necesidades de salud a pesar de su baja mortalidad es importante, pero la realidad es que falta información más amplia sobre el estado de salud de este grupo poblacional. Es necesario redefinir indicadores, mejorar los sistemas de información y reorientar la atención hacia las áreas de prevención y psicosociales. ${ }^{23}$

\section{Mortalidad}

La mayoría de las causas de muerte en adolescentes son evitables en casi $45 \%$ de los casos, debido a que desde los 10 a los 19 años de edad la población sufre riesgos adicionales quizá por la vitalidad propia de este grupo, por lo que las acciones se orientan al control de las causas más importantes, combatiendo los factores de riesgo.

En el escenario de la salud y enfermedad de México desde hace un cuarto de siglo los accidentes se ubican entre las 10 primeras causas de muerte en la población general, con una tasa en el año 2000 de 36.4 por 100000 habitantes.

En el grupo de la adolescencia temprana, de 10 a 14 años de edad, para el año 2000 se presenta como primera causa de muerte los accidentes de vehículo y de tránsito, con una tasa de 17.1 x 100000 habitantes; en segundo lugar se encuentran las agresiones y suicidios, con una tasa de 15.2; en tercer lugar los tumores, en particular las leucemias (tasa de 9.1); le siguen en tercer y cuarto lugar, respectivamente, la muerte por causas congénitas, por parálisis cerebral y por otros síndromes paralíticos y la infección respiratoria, la insuficiencia renal, la infección respiratoria aguda y las diarreas (cuadro III). ${ }^{23}$

Para 1999 el peso de los accidentes aumenta de $44 \%$ en la población de 10 a 14 años de edad, a cerca de $60 \%$ del total de defunciones del grupo de 15 a 19 años, con una tasa de 45 por cada 100,000 habitantes de este grupo de edad. Del capítulo de accidentes y agresiones, destacan en primer lugar las categorías de accidentes de transporte y agresiones.

Aunque al igual que en escolares, los accidentes son el principal daño al que está expuesta la población adolescente, la diferencia importante estriba en que en este último grupo los accidentes están determinados por las diferentes formas de violencia fuera del hogar, 
situación que se potencializa con la actitud psicológica del adolescente ante el mundo y la forma de enfrentarlo, por lo que se requiere una educación amplia y persistente en la búsqueda de modificar estilos de vida y exposición a riesgos (cuadro IV). ${ }^{24}$

Entre adolescentes el modelo de transición epidemiológica que tiene relación con los estilos de vida, el desplazamiento paulatino de las enfermedades infecciosas y parasitarias es coincidente. En estas estadísticas de mortalidad se evidencian tres fenómenos importantes para la transición epidemiológica; el primero de ellos tiene relación con la aparición de violencias, accidentes, homicidios y suicidios, efectos en la salud derivados del medio ambiente social, tránsito, urbanismo y conductas de riesgo y estilo de vida de las y los adolescentes; en el segundo hacemos énfasis en la lenta transición de las infecciones respiratorias agudas, de las diarreas y la desnutrición como problemas mencionados en las causas de mortalidad que corresponden necesariamente a aspectos sociales y económicos englobados en la pobreza; y en tercer lugar el grupo de padecimientos tumorales, congénitos, que pueden tener relación con la polución del medio ambiente.

Para el Programa Nacional para la Atención a la Salud de la Adolescencia se hace muy importante destacar éstos como capítulos a resolver en el modelo de

\section{Cuadro III}

Mortalidad general en adolescentes de 10 a 14 años de edad. República Mexicana. 1998 a 2001

\begin{tabular}{lrr} 
Causa & No & Tasa \\
Accidentes vehículo y tránsito & 1895 & 17.1 \\
\hline Agresiones y suicidios & 1683 & 15.2 \\
\hline Leucemias & 1012 & 9.1 \\
\hline Accidentes causa externa & 923 & 8.3 \\
\hline Congénitas & 586 & 5.3 \\
\hline Parálisis cerebral infantil & 462 & 4.2 \\
\hline Infecciones respiratorias agudas & 424 & 3.8 \\
\hline Insuficiencia renal crónica & 323 & 2.9 \\
\hline Desnutrición & 245 & 2.2 \\
\hline Diarreas & 236 & 2.1 \\
\hline Las 10 primeras causas & 7789 & 70.1 \\
\hline Todas las demás causas & 6980 & 62.9 \\
\hline Total & 14769 & 133.0
\end{tabular}

Total de Población de 10 a 14 a junio de $2002=11105434$

Fuente: IN EGI 2001. Mortalidad en México

\section{Cuadro IV}

Mortalidad general en adolescentes de 15 a 19 años de edad. República Mexicana. 1998 a 2001

\begin{tabular}{lrr} 
Causa & No & Tasa \\
A gresiones y suicidios & 6709 & 63.8 \\
\hline Accidentes vehículo y tránsito & 4544 & 43.2 \\
\hline Accidentes causa externa & 1521 & 14.5 \\
\hline Leucemias & 935 & 8.9 \\
\hline Maternas & 600 & 5.7 \\
\hline Agresión por medios no especificados & 536 & 5.1 \\
\hline Insuficiencia renal crónica & 529 & 5.0 \\
\hline Congénitas & 500 & 4.8 \\
\hline Infecciones respiratorias agudas & 487 & 4.6 \\
\hline Parálisis cerebral infantil & 454 & 4.3 \\
\hline Las 10 primeras causas & 16815 & 159.9 \\
\hline Todas las demás causas & 12894 & 122.6 \\
\hline Total & 29709 & 282.5
\end{tabular}

Total de Población de 10 a 14 a junio de $2002=11105434$

Fuente: IN EG I 2001. Mortalidad en México

atención, la disposición de recursos y las acciones de control..$^{25}$

\section{Discusión}

México cuenta con un bono demográfico, producto de la disminución del grupo poblacional dependiente infantil y el incremento de la población en edad productiva, que se estima reditúe en una ventana de oportunidad demográfica transitoria que se estima duraría tres décadas, después de la cual se vería reducida por un incremento en la población económicamente dependiente a expensas esta vez de la población envejecida. De tal forma el país tiene la oportunidad y el compromiso de realizar una intervención planificada e inteligente en este grupo poblacional cuyo potencial redituará en un desarrollo social y económico importante.

De no ocurrir así, la información que arrojan las diferentes fuentes sobre la situación demográfica de la adolescencia, hace tomar en cuenta dos acepciones. La primera, que puede ser positiva desde el punto de vista económico, la reducción de la proporción de la población menor de 15 años de edad, ya que potencialmente existen menos dependientes de esta edad para la población económicamente activa. La realidad es diferente pues la polaridad social y económica hace 
que el menor de 15 años de edad no solamente no dependa de un adulto sino que aquél se incorpora al mercado de trabajo en desventaja laboral. La segunda acepción es que la reducción de la proporción de adolescentes generará en los 30 años un déficit de población económicamente activa, de la que dependerán cada día más personas mayores de 64 años de edad.

Por lo tanto, el bono demográfico tiene que ser aprovechado durante las siguientes tres décadas para mejorar las condiciones socioeconómicas de la población en función de la adolescencia de hoy. ¿Qué perspectiva tenemos? A cada año que aumenta la población existe un mayor abandono escolar, un incremento del desempleo y menores oportunidades de acceso a los servicios en general. La posibilidad de que la población mejore se vuelve difícil y por lo tanto el enfoque de la transición epidemiológica debe centrarse en el momento actual en la polaridad socioeconómica, más que en la mortalidad.

Este escenario pone de relieve la necesidad de mejorar la calidad de los adultos del futuro en diferentes puntos, incrementando de su estado de salud, su capacitación y preparación, sus oportunidades de trabajo y, finalmente, su ingreso per cápita.

Adolescentes y jóvenes constituyen una fuente de riqueza invaluable y no solamente fuente de daños a la salud como calamidades sociales que hay que combatir con "buenas maneras"; la adolescencia, de manera genérica, es una esperanza social y representa el desafío de los adultos en la actualidad, pues brindarles las oportunidades que requieren para el desarrollo pleno de sus potencialidades exigirá desplegar esfuerzos importantes. Esta gran parte de la población, al igual que la sociedad, tiene características muy heterogéneas, ella, dependiendo de su origen social y de su economía actual, tiene una gama diversa de acceso a los servicios, sin embargo, el uso de los servicios depende en gran parte de la forma de consumo de su medio social. Existen desigualdades graves entre adolescentes, más acentuadas que en el resto de la población; la forma de morir y de vivir dice mucho de sus carencias y de sus necesidades.

La variedad de patrones de transición epidemiológica la podemos ver a medida que avanzamos de lo rural a lo urbano, de hombre a mujer, de niño a adolescente, con un colorido diverso y pensando siempre que ellos tienen su propia lucha en el interior de los grupos sociales y que, indefectiblemente, avanzan y llegan a pesar de todo.

Las acciones en salud tienen que orientarse en diferentes esferas, la primera de ellas es vencer las causas prevenibles con el concierto social de la respuesta organizada; la segunda, hacer atractiva la atención con un cambio de paradigma de servicios fijos a servicios que se ofrezcan en las escuelas y los centros de trabajo; el tercer nivel de respuesta es el cambio de enfoque, pasar de la simple atención a la salud a la atención enfocada no sólo a riesgo sino a resiliencia y desafío, fortalecer la economía y la producción, romper los ciclos de repetición entre las generaciones de paternidad adolescente.

\section{Conclusiones}

La aplicación de programas de salud, a través de indicadores firmes y validados, debe generar un alto desarrollo social, vencer la polaridad económica educando y previniendo el abandono escolar, así como mejorando la oferta académica en jóvenes y en adultos, vencer las barreras de género para evitar que los hombres se conviertan paulatinamente en desempleados y buscadores de violencia.

La sociedad debe pensar en la juventud como una inversión social efectiva, describir toda la escala de temas que les son interesantes para que se vean incluidos en la discusión social, que se sientan interesados en su futuro y cuidar de equilibrar los temas de su atención en el resto de la sociedad.

En este momento se debe describir la salud enfermedad de las y los adolescentes desde el enfoque de la transición epidemiológica, basados en la situación actual del proceso salud enfermedad, con la finalidad de desarrollar adecuadamente mecanismos participativos de vigilancia de la salud y de sus efectos.

El componente demográfico de la transición epidemiológica cobra especial importancia para el análisis del grupo poblacional adolescente, dado que es un factor determinante para la previsión de las acciones que deberán tomarse en el futuro no sólo para programar la atención, sino para desarrollar el potencial del capital humano que puede ser aprovechado o desaprovechado.

Dada la importancia que representa este grupo poblacional para el desarrollo del país, se hace necesario un abordaje holístico, a través del cual se haga frente a los riesgos a los que se encuentran expuestos, y que principalmente se dividen entre aquellos nuevos, propios de la modernización y urbanización a los que se encuentran sometidos, y aquellos del rezago, que aún se encuentran presentes. Es por ello que a través de este análisis se hace una invitación no solamente a continuar con la caracterización de este grupo poblacional, sino para el diseño creativo e inteligente de programas de intervención concretos que tengan 
tanto la capacidad de disminuir las amenazas como de aprovechar este potencial dinámico y creativo que caracteriza a este grupo poblacional.

\section{Referencias}

1. 0 mram AR. The epidemiologic transition theory a preliminary. J Trop Pediatr 1983;29:305-316.

2. Centro N acional para la Salud de la Infancia y laA do lescencia. Programa de Acción para laAtención a la Salud de laA dolescencia. En: Secretaría de Salud. D efiniendo las prioridades de salud para I@ s adolescentes. México, D.F.: SSA-CEN SIA, 2002.

3. Consejo $\mathrm{N}$ acional de Población. Situación actual de las y los jóvenes en México. Diagnóstico sociodemográfico. México, D.F.: C O N A PO, 2002.

4. Encuesta Nacional de Juventud 2000. Resultados Generales. México, D.F.: Secretaría de Educación Pública, Instituto Mexicano de la Juventud, agosto 2002.

5. Fondo de Población de las $\mathrm{N}$ aciones Unidas. Vivir juntos, en mundos separados. Hombres y mujeres en tiempos de cambio. Estado de la población mundial. N ueva York (N Y): FN UAP, 2000.

6. Instituto Nacional de Estadística, Geografía e Informática. VII Censo General de Población y Vivienda 1950. Resumen General. México, D.F.: IN EGI, 1952.

7. Instituto Nacional de Estadística, Geografía e Informática. VIII Censo General de Población y Vivienda 1960. Resumen General. México, D.F.: IN EGI, 1952.

8. Instituto Nacional de Estadística, Geografía e Informática. IX Censo General de Población y Vivienda 1970. Resumen General. México, D.F.: IN EGI, 1952.

9. Instituto $\mathrm{N}$ acional de Estadística, G eografía e Informática. X Censo General de Población yVivienda 1980. Resumen General. México, D.F.: IN EGI, 1952.

10. Burth M. ¿Por qué debemos invertir en el adolescente? W ashington, D.C.: Fundación W. K. Kellogg - O PS, 1998.
11. Fondo de Población de las $\mathrm{N}$ aciones Unidas. World population prospects:The 2000 Revision. N ueva York (N Y): O NU, 2000.

12. Fondo de Población de las $\mathrm{N}$ aciones U nidas. El estado de la población mundial 2001. N ueva York (N Y): FN UAP, 2001.

13. Fondo de Población de las $\mathrm{N}$ aciones Unidas. Fast facts on maternal mortality and morbidity 2002. N ueva York (N Y): FN UAP, 2002.

14. Consejo N acional de Población. Programa N acional de Población 2001 2006. México, D.F.: Conapo, diciembre 2001.

15.Villa-TL. D etección de necesidades de salud en las y los adolescentes. México, D.F.: IPAS, 2002.

16. 0 rganización Panamericana de la Salud. Perfil de salud sexual y reproductiva de los y las adolescentes y jóvenes de América Latina y El C aribe: revisión bibliográfica, 1988-1998. W ashington, D.C .: O PS, 2000.

17. Dirección General de Salud Reproductiva. La salud sexual y reproductiva de los y las adolescentes. México, D.F.: SSA-D GSR, 1999.

18. Centro $\mathrm{N}$ acional para el Control delVIH/SIDA. Programa $\mathrm{N}$ acional de Control delVIH/SIDA 2001-2006. México, D.F.: SSA, 2002.

19. G uadarrama-Rico LA. Familias mediáticas, una aproximación a las transformaciones de la clase media. En: Maldonado-Reynoso MP, ed. Horizontes comunicativos en México. Estudios Críticos. México, D.F.:A sociación Mexicana de Investigadores Comunicadores, 2002.

20. Consejo $\mathrm{N}$ acional contra las Adicciones. Encuesta $\mathrm{N}$ acional de Adicciones. 1998. México, D.F.: Secretaría de Salud, 2000.0

21. Borges G, Rosovsky H, Gómez C, G utiérrez R. Epidemiology of suicide in Mexico from 1970 to 1994. Salud Publica Mex 1996;38:197-206.

22. Rivera-D ommarco J, Shamah-Levy T,Villalpando-H ernández S, González de Cossío T, Hernández-Prado B, Sepúlveda J. Encuesta N acional de Nutrición 2000. Instituto N acional de Salud Pública. México, D.F.: Secretaría de Salud, 2001.

23. Dirección General de Epidemiología. Sistema de Vigilancia Epidemiológica. Secretaría de Salud, México, D.F.: 2001.

24. Consejo $\mathrm{N}$ acional para la Prevención de Accidentes. Programa N acional para la Prevención y Control de Accidentes. 2001-2006. México, D.F.: SSA, 2002.

25. Instituto $N$ acional de Estadística, Geografía e Informática. D años a la salud. Tabulados Básicos y por Entidad. México, D.F. IN EGI, 2000. 